\title{
Article
}

\section{New Bounds for the Sine Function and Tangent Function}

\author{
Ling Zhu
}

Abstract: Using the power series expansion technique, this paper established two new inequalities for the sine function and tangent function bounded by the functions $x^{2}(\sin (\lambda x) /(\lambda x))^{\alpha}$ and $x^{2}(\tan (\mu x) /(\mu x))^{\beta}$. These results are better than the ones in the previous literature.

Keywords: Mitrinović-Adamović-type inequality; Becker-Stark-type inequality; circular functions

\section{Introduction}

Because of the fact the functions $\cos x$ and $(\sin x) / x$ are less than 1 for $x \in(0, \pi / 2)$, in order to determine this relationship $(\sin x) / x$ and the weighted geometric mean of $\cos x$ and 1, we examine the Taylor expansion of the following function:

$$
\begin{aligned}
\frac{\sin x}{x}-(\cos x)^{\beta}= & \left(\frac{1}{2} \beta-\frac{1}{6}\right) x^{2}+\left(-\frac{1}{8} \beta^{2}+\frac{1}{12} \beta+\frac{1}{120}\right) x^{4} \\
& +\left(\frac{1}{48} \beta^{3}-\frac{1}{24} \beta^{2}+\frac{1}{45} \beta-\frac{1}{5040}\right) x^{6}+O\left(x^{8}\right) .
\end{aligned}
$$

When choosing $\beta=1 / 3$ in above formula we can obtain the following fact

$$
\frac{\sin x}{x}-(\cos x)^{1 / 3}=\frac{1}{45} x^{4}+\frac{19}{5670} x^{6}+O\left(x^{8}\right),
$$

which will motivate us to prove the following inequality

$$
\frac{\sin x}{x}>(\cos x)^{1 / 3}
$$

holds for $0<x<\pi / 2$. The above inequality was confirmed by Mitrinović and Adamović in [1], so we call it Mitrinović-Adamović inequality. On the other hand, the relationship between $(\sin x) / x$ and the weighted arithmetic mean of $\cos x$ and 1 has been discussed in Zhu [2] just published, described as the following inequality similarly:

$$
\frac{\sin x}{x}<\frac{2}{3}+\frac{1}{3} \cos x
$$

or

$$
\frac{3 \sin x}{2+\cos x}<x
$$

In 1451, using a geometrical method Nicolaus De Cusa (1401-1464) discovered (3), and in 1664 when considering the estimation of $\pi$ Christian Huygens (1629-1695) confirmed (2). In view of the above historical facts (see [3-10]), we call the inequality (2) Cusa-Huygens inequality.

In 2018, Zhu [11] shown two improvements to (3) as follows: the inequalities

$$
\frac{1}{180} x^{5}<x-\frac{3 \sin x}{2+\cos x}
$$


and

$$
\frac{1}{2100} x^{7}<x-\frac{3 \sin x}{2+\cos x}\left[1+\frac{(1-\cos x)^{2}}{9(3+2 \cos x)}\right]
$$

hold for all $x \in(0, \pi]$, where $1 / 180$ and $1 / 2100$ are the best constants in previous inequalities, respectively. Two results of previous proposition are corrections of Theorem 3.4.20 from monograph Mitrinović [9]. Malešević et al. [12] made a bilateral supplement to the above two inequalities. Chen and Cheung [13] obtained the bounds for $(\sin x) / x$ in term of $((2+\cos x) / 3)^{\delta}$ as follows

$$
\left(\frac{2+\cos x}{3}\right)^{\theta_{0}}<\frac{\sin x}{x}<\left(\frac{2+\cos x}{3}\right)^{\vartheta_{0}}
$$

holds for all $0<x<\pi / 2$, where $\vartheta_{0}=1$ and $\theta_{0}=(\ln \pi-\ln 2) /(\ln 3-\ln 2)$ are the best possible constants in (6). The double inequality (6) was proved by Bagul [14] and Zhu [15] in different ways. In Zhu [15] some new improvements to inequality (2) can be found:

$$
\begin{gathered}
\left(1-\frac{x^{3}}{\pi^{3}}\right) \frac{2+\cos x}{3}<\frac{\sin x}{x}<\left(1-\frac{x^{4}}{180}\right) \frac{\cos x+2}{3} \\
{\left[1+\frac{8(\pi-3)}{\pi^{3}} x^{2}\right] \frac{2+\cos x}{3}-\frac{8(\pi-3)}{\pi^{3}} x^{2}<\frac{\sin x}{x}<\left(1+\frac{1}{30} x^{2}\right) \frac{2+\cos x}{3}-\frac{1}{30} x^{2},}
\end{gathered}
$$

and

$$
\begin{aligned}
& {\left[1+\frac{1}{30} x^{2}+\frac{2\left(240 \pi-\pi^{3}-720\right)}{15 \pi^{5}} x^{4}\right] \frac{2+\cos x}{3}-\left[\frac{1}{30} x^{2}+\frac{2\left(240 \pi-\pi^{3}-720\right)}{15 \pi^{5}} x^{4}\right] } \\
< & \frac{\sin x}{x} \\
< & \left(1+\frac{1}{30} x^{2}+\frac{1}{840} x^{4}\right) \frac{2+\cos x}{3}-\left(\frac{1}{30} x^{2}+\frac{1}{840} x^{4}\right)
\end{aligned}
$$

hold for $0<x<\pi / 2$.

Bercu [16] used the truncations of Fourier cosine series to the inequality (2) and obtained an enhanced form of (2):

$$
\frac{\sin x}{x}-\frac{2+\cos x}{3}<-\frac{1}{45}(1-\cos x)^{2}, 0<x<\frac{\pi}{2} .
$$

Bagul et al. [17] draw two conclusions about the improvement of inequality (2):

$$
-\left(\frac{2}{3}-\frac{2}{\pi}\right) \frac{1}{(\pi / 2-1)}(x-\sin x)<\frac{\sin x}{x}-\frac{2+\cos x}{3}<-\left(\frac{2}{3}-\frac{2}{\pi}\right) \frac{1}{(\pi / 2-1)^{2}}(x-\sin x)^{2}
$$

and

$$
-\left(\frac{2}{3}-\frac{2}{\pi}\right)(\sin x-x \cos x)<\frac{\sin x}{x}-\frac{2+\cos x}{3}<-\left(\frac{2}{3}-\frac{2}{\pi}\right)(\sin x-x \cos x)^{2}
$$

hold for $0<x<\pi / 2$.

Recently, Zhu [2] improved the famous inequality (2) using two different technology paths and draw two results as follows: for $0<x<\pi / 2$, the two inequalities

$$
\frac{\sin x}{x}-\frac{2+\cos x}{3}<-\frac{1}{180} x^{4}\left(\frac{\sin x}{x}\right)^{2 / 7}
$$


and

$$
\frac{\sin x}{x}-\frac{2+\cos x}{3}<-\left(\frac{2}{3}-\frac{2}{\pi}\right)(\sin x-x \cos x)^{\left(\pi^{2}-12\right) /\left(3 \pi^{2}-\pi^{3}\right)}
$$

hold with the best constant $-1 / 180$ and $(2 / 3-2 / \pi)$ respectively.

Inequalities on two functions $(\sin x) / x$ and $(\tan x) / x$ arouse great enthusiasm of researchers. Interested readers can refer to these literatures [18-61] and monograph [62] which was edited by Rassias and Andrica.

This paper focuses on some new bounds for the functions $\sin (x) / x$ and $\tan (x) / x$ and wants to improve the following inequalities:

$$
1-\frac{\sin x}{x}>0 \text { and } \frac{\tan x}{x}-1>0
$$

Recently, Wu and Bercu [63] thought of Fourier series technology to approximate these two functions. They considered the power series expansion of the following function

$$
\begin{aligned}
& 1-\frac{\sin x}{x}-(a+b \cos x+c \cos 2 x+d \cos 3 x) \\
= & -(a+b+c+d)+x^{2}\left(\frac{1}{2} b+2 c+\frac{9}{2} d+\frac{1}{6}\right)-x^{4}\left(\frac{1}{24} b+\frac{2}{3} c+\frac{27}{8} d+\frac{1}{120}\right) \\
& +x^{6}\left(\frac{1}{720} b+\frac{4}{45} c+\frac{81}{80} d+\frac{1}{5040}\right)+O\left(x^{8}\right) .
\end{aligned}
$$

To obtain a slightly higher precision approximation, they let

$$
\left\{\begin{array}{ccc}
a+b+c+d & = & 0 \\
\frac{1}{2} b+2 c+\frac{9}{2} d+\frac{1}{6} & =0 \\
\frac{1}{24} b+\frac{2}{3} c+\frac{27}{8} d+\frac{1}{120} & =0 \\
\frac{1}{720} b+\frac{4}{45} c+\frac{81}{80} d+\frac{1}{5040} & =0
\end{array}\right.
$$

to obtain these constants

$$
a=\frac{359}{945}, b=-\frac{167}{420}, c=\frac{2}{105}, d=-\frac{1}{756}
$$

and find

$$
1-\frac{\sin x}{x}-\left(\frac{359}{945}-\frac{167}{420} \cos x+\frac{2}{105} \cos 2 x-\frac{1}{756} \cos 3 x\right)=\frac{23}{226800} x^{8}+O\left(x^{10}\right)
$$

which leads them to prove

$$
\begin{aligned}
1-\frac{\sin x}{x} & >\frac{359}{945}-\frac{167}{420} \cos x+\frac{2}{105} \cos 2 x-\frac{1}{756} \cos 3 x \\
& =\frac{(1-\cos x)\left(-31 \cos x+5 \cos ^{2} x+341\right)}{945}
\end{aligned}
$$

This technique can be used to deal with the approximation problem of another function $\tan (x) / x-1$, and then they obtain the following results. 
Proposition 1 ([63]). The following inequalities

$$
\begin{aligned}
& 1-\frac{\sin x}{x}>\frac{(1-\cos x)\left(-31 \cos x+5 \cos ^{2} x+341\right)}{945}, \\
& \frac{\tan x}{x}-1>\frac{(1-\cos x)\left(604 \cos ^{2} x-1817 \cos x+1843\right)}{945}, \\
& 1-\frac{\sin x}{x}>1-\left(\frac{2+\cos x}{3}-\frac{1}{180} x^{4}+\frac{1}{3780} x^{6}\right) \\
&>1-\left(1+\frac{(1-\cos x)\left(-5 \cos ^{2} x+31 \cos x-341\right)}{945}\right) \\
&>1-\frac{2+\cos x}{3}
\end{aligned}
$$

hold for all $x \in(0, \pi / 2)$.

In this paper, we want to obtain an approximation with appropriate accuracy about these two functions. We examine the power series expansion of function in the following form

$$
\begin{aligned}
& 1-\frac{\sin x}{x}-a x^{2}\left(\frac{\sin b x}{b x}\right)^{c} \\
= & -x^{2}\left(a-\frac{1}{6}\right)+x^{4}\left(\frac{1}{6} a b^{2} c-\frac{1}{120}\right)+x^{6}\left[a\left(\frac{1}{180} b^{4} c-\frac{1}{72} b^{4} c^{2}\right)+\frac{1}{5040}\right] \\
& +x^{8}\left[\frac{1}{45360} a b^{6} c\left(35 c^{2}-42 c+16\right)-\frac{1}{362880}\right]+O\left(x^{10}\right),
\end{aligned}
$$

and let

$$
\left\{\begin{array}{cl}
a-\frac{1}{6} & =0 \\
\frac{1}{6} a b^{2} c-\frac{1}{120} & =0 \\
a\left(\frac{1}{180} b^{4} c-\frac{1}{72} b^{4} c^{2}\right)+\frac{1}{5040} & =0
\end{array}\right.
$$

to determine

$$
a=\frac{1}{6}, b= \pm \frac{\sqrt{7}}{14}, c=\frac{42}{5}
$$

We can obtain that

$$
1-\frac{\sin x}{x}-\frac{1}{6} x^{2}\left(\frac{\sin \frac{\sqrt{7}}{14} x}{\frac{\sqrt{7}}{14} x}\right)^{\frac{42}{5}}=\frac{1}{4116000} x^{8}+O\left(x^{10}\right)
$$

In the same way, we obtain

$$
\frac{\tan x}{x}-1-\frac{1}{3} x^{2}\left(\frac{\tan \frac{\sqrt{43}}{7} x}{\frac{\sqrt{43}}{7} x}\right)^{\frac{294}{215}}=\frac{12416}{18907875} x^{8}+O\left(x^{10}\right) .
$$

With the above foreshadowing, we can now announce the main work of this paper which established two inequalities of exponential type for the functions $1-(\sin x) / x$ and $(\tan x) / x-1$ bounded by the function $x^{2}(\sin (\lambda x) /(\lambda x))^{\alpha}$ and $x^{2}(\tan (\mu x) /(\mu x))^{\beta}$ as follows. 
Theorem 1. Let $0<|x|<\pi / 2, \phi=1$ and

$$
\varphi=\left[\frac{24^{5} \pi^{27}(\pi-2)^{5}}{4^{42} 7^{21}\left(\sin \frac{\sqrt{7}}{28} \pi\right)^{42}}\right]^{1 / 5}>1
$$

Then the double inequality

$$
\phi\left[\frac{1}{6} x^{2}\left(\frac{\sin \frac{1}{2 \sqrt{7}} x}{\frac{1}{2 \sqrt{7}} x}\right)^{\frac{42}{5}}\right]<1-\frac{\sin x}{x}<\varphi\left[\frac{1}{6} x^{2}\left(\frac{\sin \frac{1}{2 \sqrt{7}} x}{\frac{1}{2 \sqrt{7}} x}\right)^{\frac{42}{5}}\right]
$$

holds with the best constants $\phi$ and $\varphi$.

Theorem 2. Let $0<|x|<\pi / 2$. Then

$$
\frac{\tan x}{x}-1>\frac{1}{3} x^{2}\left(\frac{\tan \frac{\sqrt{43}}{7} x}{\frac{\sqrt{43}}{7} x}\right)^{\frac{294}{215}}
$$

holds with the best constant $1 / 3$.

\section{Lemmas}

The proof of the main conclusions (Theorems 1 and 2) of this paper needs the following lemmas as the basis.

Lemma 1. Let $n \geq 3, n \in \mathbb{N}$,

$$
\begin{aligned}
& \sigma_{1}=\frac{\sqrt{7}+14}{14} \approx 1.1890, \\
& \sigma_{2}=\frac{14-\sqrt{7}}{14} \approx 0.81102, \\
& \sigma_{3}=\frac{\sqrt{7}}{14} \approx 0.18898,
\end{aligned}
$$

and for $k \geq 4$,

$$
\begin{aligned}
a_{k}= & -\frac{27}{2} \sqrt{7} \frac{\sigma_{1}^{2 k+2}}{(2 k+2) !}+\frac{27}{2} \sqrt{7} \frac{\sigma_{2}^{2 k+2}}{(2 k+2) !}-21 \frac{\sigma_{3}^{2 k}}{(2 k) !} \\
& +32 \sqrt{7} \frac{\sigma_{3}^{2 k+1}}{(2 k+1) !}+\frac{5 \sqrt{7}+21}{2} \frac{\sigma_{2}^{2 k+1}}{(2 k+1) !}+\frac{21-5 \sqrt{7}}{2} \frac{\sigma_{1}^{2 k+1}}{(2 k+1) !} .
\end{aligned}
$$

Then $2 a_{2 n}-5 a_{2 n+1}>0$.

Proof. Since

$$
\begin{aligned}
a_{2 n}= & -\frac{27}{2} \sqrt{7} \frac{\sigma_{1}^{4 n+2}}{(4 n+2) !}+\frac{27}{2} \sqrt{7} \frac{\sigma_{2}^{4 n+2}}{(4 n+2) !}-21 \frac{\sigma_{3}^{4 n}}{(4 n) !} \\
& +32 \sqrt{7} \frac{\sigma_{3}^{4 n+1}}{(4 n+1) !}+\frac{5 \sqrt{7}+21}{2} \frac{\sigma_{2}^{4 n+1}}{(4 n+1) !}+\frac{21-5 \sqrt{7}}{2} \frac{\sigma_{1}^{4 n+1}}{(4 n+1) !}, \\
a_{2 n+1}= & -\frac{27}{2} \sqrt{7} \frac{\sigma_{1}^{4 n+4}}{(4 n+4) !}+\frac{27}{2} \sqrt{7} \frac{\sigma_{2}^{4 n+4}}{(4 n+4) !}-21 \frac{\sigma_{3}^{4 n+2}}{(4 n+2) !} \\
& +32 \sqrt{7} \frac{\sigma_{3}^{4 n+3}}{(4 n+3) !}+\frac{5 \sqrt{7}+21}{2} \frac{\sigma_{2}^{4 n+3}}{(4 n+3) !}+\frac{21-5 \sqrt{7}}{2} \frac{\sigma_{1}^{4 n+3}}{(4 n+3) !},
\end{aligned}
$$


we compute to obtain

$$
(4 n+4) !\left(2 a_{2 n}-5 a_{2 n+1}\right)=\left[\frac{5}{2} u(n) \sigma_{1}^{4 n+1}-512 v(n) \sigma_{3}^{4 n}\right]+\frac{5}{2} w(n) \sigma_{2}^{4 n+1},
$$

where

$$
\begin{aligned}
u(n)= & \left(\frac{2688}{5}-128 \sqrt{7}\right) n^{3}-\left(\frac{2304}{5} \sqrt{7}-\frac{5616}{5}\right) n^{2}-\left(\frac{17559}{35} \sqrt{7}-\frac{3277}{5}\right) n \\
& -\left(\frac{19459}{140} \sqrt{7}-\frac{31019}{280}\right) \\
v(n)= & (n+1)\left(21 n^{3}+\frac{55}{2} n^{2}+\frac{1193}{128} n+\frac{1445}{3584}\right), \\
w(n)= & \left(128 \sqrt{7}+\frac{2688}{5}\right) n^{3}+\left(\frac{2304}{5} \sqrt{7}+\frac{5616}{5}\right) n^{2}+\left(\frac{17559}{35} \sqrt{7}+\frac{3277}{5}\right) n \\
& +\left(\frac{14436973}{109760} \sqrt{7}+\frac{75065}{784}\right)
\end{aligned}
$$

are positive for $n \geq 3$. In order to prove Lemma 1, it suffices to prove that for $n \geq 3$,

$$
\frac{5}{2} u(n) \sigma_{1}^{4 n+1}-512 v(n) \sigma_{3}^{4 n}>0 \Longleftrightarrow\left(\frac{\sigma_{1}}{\sigma_{3}}\right)^{4 n}>\frac{1024}{5 \sigma_{1}} \frac{v(n)}{u(n)} .
$$

To note the fact

$$
\frac{\sigma_{1}}{\sigma_{3}}=1+2 \sqrt{7} \approx 6.2915>6,
$$

we only need to prove

$$
6^{4 n}>\frac{1024}{5 \sigma_{1}} \frac{v(n)}{u(n)}
$$

By mathematical induction, we can prove the inequality (23). First, the inequality (23) is obviously true for $n=3$. Assume that (23) holds for $n=m \geq 3$, that is,

$$
6^{4 m}>\frac{1024}{5 \sigma_{1}} \frac{v(m)}{u(m)}
$$

holds. In the following, we shall prove that (23) holds for $n=m+1$. Since

$$
6^{4(m+1)}=6^{4} \cdot 6^{4 m}>1296\left[\frac{1024}{5 \sigma_{1}} \frac{v(m)}{u(m)}\right],
$$

we can complete the proof of (23) when showing that

$$
1296\left[\frac{1024}{5 \sigma_{1}} \frac{v(m)}{u(m)}\right]>\frac{1024}{5 \sigma_{1}} \frac{v(m+1)}{u(m+1)},
$$

or

$$
\frac{A}{B}:=\frac{1296 v(m)}{u(m)}>\frac{v(m+1)}{u(m+1)}:=\frac{C}{D}
$$


In fact,

$$
\begin{aligned}
A D-B C= & 1296 v(m) u(m+1)-v(m+1) u(m) \\
= & \left(\frac{48280304638987025}{200704}-\frac{7757241843454045}{100352} \sqrt{7}\right) \\
& +\left(\frac{12227207591977687}{28672}-\frac{1898338908715519}{14336} \sqrt{7}\right)(m-3) \\
& +\left(\frac{11559549671546923}{35840}-\frac{10809910365147}{112} \sqrt{7}\right)(m-3)^{2} \\
& +\left(\frac{17268465300163}{128}-\frac{24831245768359}{640} \sqrt{7}\right)(m-3)^{3} \\
& +\left(\frac{674131077463}{20}-\frac{18578001121}{2} \sqrt{7}\right)(m-3)^{4} \\
& +\left(5030226642-\frac{6621922062}{5} \sqrt{7}\right)(m-3)^{5} \\
& +\left(\frac{2075901072}{5}-104111168 \sqrt{7}\right)(m-3)^{6} \\
& +(14620032-3480960 \sqrt{7})(m-3)^{7} \\
> & 0
\end{aligned}
$$

holds for all $m \geq 3$. This completes the proof of Lemma 1 .

It is not difficult to prove the following conclusion in the similar way.

Lemma 2. Let $n \geq 2, n \in \mathbb{N}$,

$$
\begin{aligned}
& \rho_{1}=\frac{2}{7} \sqrt{43}+2 \approx 3.8736 \\
& \rho_{2}=\frac{2}{7} \sqrt{43} \approx 1.8736, \\
& \rho_{3}=2-\frac{2}{7} \sqrt{43} \approx 0.12645,
\end{aligned}
$$

and for $k \geq 4$,

$$
\begin{aligned}
b_{k}= & \frac{17 \sqrt{43}}{903 \cdot 2} \frac{\rho_{1}^{2 k+1}}{(2 k+1) !}-\frac{117 \sqrt{43}}{4816} \frac{\rho_{1}^{2 k+2}}{(2 k+2) !} \\
& +\frac{1}{2} \frac{2^{2 k}}{(2 k) !}-\frac{1}{2} \frac{2^{2 k+1}}{(2 k+1) !} \\
& +\frac{283 \sqrt{43}}{3612} \frac{\rho_{2}^{2 k+1}}{(2 k+1) !}-\frac{17 \sqrt{43}}{903 \cdot 2} \frac{\rho_{3}^{2 k+1}}{(2 k+1) !}+\frac{117 \sqrt{43}}{4816} \frac{\rho_{3}^{2 k+2}}{(2 k+2) !} .
\end{aligned}
$$

Then $2 b_{2 n}-5 b_{2 n+2}>0$.

\section{Proofs of Theorems 1 and 2}

Proof of Theorem 1. Let

$$
F(x)=\ln \left(1-\frac{\sin x}{x}\right)-\ln \left[\frac{1}{6} x^{2}\left(\frac{\sin \frac{1}{2 \sqrt{7}} x}{\frac{1}{2 \sqrt{7}} x}\right)^{\frac{42}{5}}\right],
$$


where $0<|x|<\pi / 2$. Since this function $F(x)$ is even, let's consider the problem on interval $(0, \pi / 2)$. We compute to obtain

$$
F^{\prime}(x)=\frac{2 \sqrt{7}}{35} \frac{f(x)}{x\left(\cos \sigma_{1} x-\cos \sigma_{2} x+2 x \sin \sigma_{3} x\right)},
$$

where

$$
\begin{aligned}
f(x)= & \frac{27}{2} \sqrt{7} \cos \sigma_{1} x-\frac{27}{2} \sqrt{7} \cos \sigma_{2} x-21 x^{2} \cos \sigma_{3} x+32 \sqrt{7} x \sin \sigma_{3} x \\
& +\left(\frac{5}{2} \sqrt{7}+\frac{21}{2}\right) x\left(\sin \sigma_{2} x\right)-\left(\frac{5}{2} \sqrt{7}-\frac{21}{2}\right) x\left(\sin \sigma_{1} x\right) .
\end{aligned}
$$

and $\sigma_{i}(i=1,2,3)$ are defined as (21). Substituting

$$
\sin x=\sum_{k=0}^{\infty} \frac{(-1)^{k}}{(2 k+1) !} x^{2 k+1}, \cos x=\sum_{k=0}^{\infty} \frac{(-1)^{k}}{(2 k) !} x^{2 k}
$$

into (26), we can obtain that

$$
\begin{aligned}
f(x):= & \sum_{k=4}^{\infty}(-1)^{k} a_{k} x^{2 k+2}=\sum_{n=2}^{\infty}\left(a_{2 n} x^{4 n+2}-a_{2 n+1} x^{4 n+4}\right) \\
= & \left(\frac{1}{274400} x^{10}-\frac{163}{1521273600} x^{12}\right)+\sum_{n=3}^{\infty}\left(a_{2 n} x^{4 n+2}-a_{2 n+1} x^{4 n+4}\right),
\end{aligned}
$$

where $a_{k}$ is defined by (22). Since

$$
\frac{1}{274400} x^{10}-\frac{163}{1521273600} x^{12}>0
$$

for all $x \in(0, \pi / 2)$, we can determine the positive definiteness of the function $f(x)$ on $(0, \pi / 2)$ when proving

$$
a_{2 n} x^{4 n+2}-a_{2 n+1} x^{4 n+4}>0 \Longleftrightarrow x^{2}<\frac{a_{2 n}}{a_{2 n+1}}
$$

for $n \geq 3$. Since

$$
x^{2}<\left(\frac{\pi}{2}\right)^{2} \approx 2.4674<\frac{5}{2}
$$

we can prove (28) when proving that for $n \geq 3$,

$$
\frac{5}{2}<\frac{a_{2 n}}{a_{2 n+1}}
$$

or

$$
2 a_{2 n}-5 a_{2 n+1}>0,
$$

which comes from Lemma 1.

So $f(x)>0$ and $F(x)$ is increasing on $(0, \pi / 2)$. In view of

$$
F\left(0^{+}\right)=0, F\left(1^{-}\right)=\frac{1}{5} \ln \left(\frac{24^{5} \pi^{27}(\pi-2)^{5}}{4^{42} 7^{21}\left(\sin \frac{\sqrt{7}}{28} \pi\right)^{42}}\right)>0,
$$

the proof of Theorem 1 is complete. 
Proof of Theorem 2. Let

$$
G(x)=\ln \left(\frac{\tan x}{x}-1\right)-\ln \left[\frac{1}{3} x^{2}\left(\frac{\tan \frac{\sqrt{43}}{7} x}{\frac{\sqrt{43}}{7} x}\right)^{\frac{294}{215}}\right], 0<x<\frac{\pi}{2} .
$$

Then

$$
G^{\prime}(x)=\frac{1806 \sqrt{43}}{9245} \frac{g(x)}{x\left(\tan \frac{\sqrt{43}}{7} x\right)(\tan x-x) \cos ^{2} \frac{\sqrt{43}}{7} x \cos ^{2} x}
$$

where

$$
\begin{aligned}
g(x)= & \frac{117 \sqrt{43}}{4816} \cos \rho_{1} x-\frac{117 \sqrt{43}}{4816} \cos \rho_{3} x \\
& -\frac{1}{2} x \sin 2 x+\frac{1}{2} x^{2} \cos 2 x+\frac{1}{2} x^{2} \\
& +\frac{283 \sqrt{43}}{3612} x \sin \rho_{2} x+\frac{17 \sqrt{43}}{903 \cdot 2} x \sin \rho_{1} x-\frac{17 \sqrt{43}}{903 \cdot 2} x \sin \rho_{3} x,
\end{aligned}
$$

where $\rho_{i}(i=1,2,3)$ are defined as (24). Substituting (27) into (29), we can obtain that

$$
g(x)=: \sum_{k=4}^{\infty}(-1)^{k} b_{k} x^{2 k+2}
$$

where $b_{k}$ is defined by (25). We can rewrite $g(x)$ as

$$
g(x)=\sum_{n=2}^{\infty}\left(b_{2 n} x^{4 n+2}-b_{2 n+1} x^{4 n+4}\right) .
$$

Then, we determine the positive definiteness of the function $g(x)$ on $(0, \pi / 2)$ when proving

$$
b_{2 n} x^{4 n+2}-b_{2 n+1} x^{4 n+4}>0 \Longleftrightarrow x^{2}<\frac{b_{2 n}}{b_{2 n+1}}
$$

for $n \geq 3$. Since

$$
x^{2}<\left(\frac{\pi}{2}\right)^{2}=\frac{\pi^{2}}{4} \approx 2.4674<\frac{5}{2},
$$

we can prove (30) when proving that for $n \geq 3$,

$$
\frac{5}{2}<\frac{b_{2 n}}{b_{2 n+1}}
$$

which is the result of Lemma 2. So $g(x)>0$ and $G(x)$ is strictly increasing on $(0, \pi / 2)$. Therefore $G(x)>G\left(0^{+}\right)=0$. Considering the reason

$$
\lim _{x \rightarrow 0^{+}} \frac{\frac{\tan x}{x}-1}{x^{2}\left(\frac{\tan \frac{\sqrt{43}}{7} x}{\frac{\sqrt{43}}{7} x}\right)^{\frac{294}{215}}}=\frac{1}{3}
$$

the proof of Theorem 2 is completed.

\section{Comparison of New and Old Results}

When letting $\phi=1$ in (19) we can obtain

$$
1-\frac{\sin x}{x}>\frac{1}{6} x^{2}\left(\frac{\sin \frac{1}{2 \sqrt{7}} x}{\frac{1}{2 \sqrt{7}} x}\right)^{\frac{42}{5}}
$$


By using the similar proof method of Theorem 1 it is not difficult to prove the following results:

$$
\begin{aligned}
& \frac{1}{6} x^{2}\left(\frac{\sin \frac{1}{2 \sqrt{7}} x}{\frac{1}{2 \sqrt{7}} x}\right)^{\frac{42}{5}}>1-\left(\frac{2+\cos x}{3}-\frac{1}{180} x^{4}+\frac{1}{3780} x^{6}\right) \\
& \frac{1}{3} x^{2}\left(\frac{\tan \frac{\sqrt{43}}{7} x}{\frac{\sqrt{43}}{7} x}\right)^{\frac{294}{215}}>\frac{(1-\cos x)\left(604 \cos ^{2} x-1817 \cos x+1843\right)}{945}
\end{aligned}
$$

hold for all $x \in(0, \pi / 2)$. So new results (19) and (20) are better that the old ones (16) and (17), respectively. In addition, there are deeper conclusions:

$$
\begin{aligned}
1-\frac{\sin x}{x} & >\frac{1}{6} x^{2}\left(\frac{\sin \frac{1}{2 \sqrt{7}} x}{\frac{1}{2 \sqrt{7}} x}\right)^{\frac{42}{5}} \\
& >1-\left(\frac{2+\cos x}{3}-\frac{1}{180} x^{4}+\frac{1}{3780} x^{6}\right) \\
& >\frac{(1-\cos x)\left(5 \cos ^{2} x-31 \cos x+341\right)}{945}>1-\frac{2+\cos x}{3} .
\end{aligned}
$$

Funding: This paper is supported by the Natural Science Foundation of China grants No. 61772025.

Acknowledgments: The author is thankful to reviewers for reviewers' careful corrections to and valuable comments on the original version of this paper. This paper is supported by the Natural Science Foundation of China (No. 61772025).

Conflicts of Interest: The author declares that he has no conflict of interest.

\section{References}

1. Mitrinović, D.S.; Adamović, D.D. Sur une inégalité élémentaire où interviennent des fonctions trigonométriques. Publ. Elektroteh. Fak. Ser. Mat. Fiz. 1965, 143-155, 23-34.

2. Zhu, L. New Inequalities of Cusa-Huygens Type. Mathematics 2021, 9, 2101. [CrossRef]

3. Campan, F.T. The Story of Number $\pi$; Editura Albatros: Bucuresti, Romania, 1977.

4. Iuskevici, A.P. History of Mathematics in 16th and 16th Centuries; National Publishing House: Moscow, Russia, 1961.

5. Cajori, F. A History of Mathematics; MacMillan and Co.: New York, NY, USA; London, UK, 1894.

6. Huygens, C. Oeuvres Completes, Publiees par la Societe Hollandaise des Science; M. Nijhoff: Haga, Sweden, 1988; Volume 20.

7. Queries-Replies. Math. Comput. 1949, 3, 561-563. [CrossRef]

8. Vahlen, K.T. Konstruktionen und Approximationen in Systematischer Darstellung; BG Teubner: Leipzig, Germany, 1911; pp. 188-190.

9. Mitrinović, D.S. Analytic Inequalities; Springer: Berlin/Heidelberg, Germany, 1970.

10. Sándor, J.; Bencze, M. On Huygens' trigonometric inequality. RGMIA Res. Rep. Collect. 2005, 8, 1-4.

11. Zhu, L. On Frame's inequalities. J. Inequal. Appl. 2018, 2018, 94. [CrossRef]

12. Malesevic, B.; Nenezic, M.; Zhu, L.; Banjac, B.; Petrovic, M. Some new estimates of precision of Cusa-Huygens and Huygens approximations. Appl. Anal. Discret. Math. 2021, 15, 243-259. [CrossRef]

13. Chen, C.-P.; Cheung, W.-S. Sharp Cusa and Becker-Stark inequalities. J. Inequal. Appl. 2011, 2011, 136. [CrossRef]

14. Bagul, Y.J. Remark on the paper of Zheng Jie Sun and Ling Zhu. J. Math. Inequal. 2019, 13, 801-803. [CrossRef]

15. Zhu, L. New Cusa-Huygens type inequalities. AIMS Math. 2020, 5, 4874-4888. [CrossRef]

16. Bercu, G. Fourier series method related to Wilker-Cusa-Huygens inequalities. Math. Inequal. Appl. 2019, 22, 1091-1098. [CrossRef]

17. Bagul, Y.J.; Banjac, B.; Chesneau, C.; Kostic, M.; Malesević, B. New refinements of Cusa-Huygens inequality. Results Math. 2021, 76, 107. [CrossRef]

18. Zhu, L. A source of inequalities for circular functions. Comput. Math. Appl. 2009, 58, 1998-2004. [CrossRef]

19. Mortici, C. The natural approach of Wilker-Cusa-Huygens inequalities. Math. Inequal. Appl. 2011, 14, 535-541. [CrossRef]

20. Chen, X.-D.; Shi, J.; Wang, Y.; Xiang, P. A New Method for Sharpening the Bounds of Several Special Functions. Results Math. 2017, 72, 695-702. [CrossRef]

21. Chen, X.-D.; Ma, J.; Jin, J.; Wang, Y. A two-point-Pade-approximant-based method for bounding some trigonometric functions. J. Inequal. Appl. 2018, 2018, 140. [CrossRef] 
22. Chen, X.-D.; Ma, J.; Li, Y. Approximating trigonometric functions by using exponential inequalities. J. Inequal. Appl. 2019, 2019, 53. [CrossRef]

23. Banjac, B. System for Automatic Proving of Some Classes of Analytic Inequalities. Ph.D. Thesis, School of Electrical Engineering, Belgrade, Serbia, May 2019. Available online: http:/ / nardus.mpn.gov.rs/ (accessed on 17 July 2021). (In Serbian)

24. Wang, M.-K.; Wang, Z.-K.; Chu, Y.-M. An optimal double inequality between geometric and identric means. Appl. Math. Lett. 2012, 25, 471-475. [CrossRef]

25. Qiu, Y.-F.; Wang, M.-K.; Chu, Y.-M.; Wang, G.-D. Two sharp inequalities for Lehmer mean, identric mean and logarithmic mean. J. Math. Inequal. 2011, 5, 301-306. [CrossRef]

26. Chu, Y.-M.; Long, B.-Y. Sharp inequalities between means. Math. Inequal. Appl. 2011, 14, 647-655. [CrossRef]

27. Wang, M.-K.; Hong, M.-Y.; Xu, Y.-F.; Shen, Z.-H.; Chu, Y. Inequalities for generalized trigonometric and hyperbolic functions with one parameter. J. Math. Inequal. 2020, 14, 1-21. [CrossRef]

28. Lv, Y.-P.; Wang, G.-D.; Chu, Y.-M. A note on Jordan type inequalities for hyperbolic functions. Appl. Math. Lett. 2012, 25, 505-508. [CrossRef]

29. Neuman, E.; Sándor, J. On some inequalities involving trigonometric and hyperbolic functions with emphasis on the CusaHuygens, Wilker, and Huygens inequalities. Math. Inequal. Appl. 2010, 13, 715-723. [CrossRef]

30. Zhu, L. Some new inequalities of the Huygens type. Comput. Math. Appl. 2009, 58, 1180-1182. [CrossRef]

31. Bercu, G. Padé approximant related to remarkable inequalities involving trigonometric functions. J. Inequal. Appl. 2016, 2016, 99. [CrossRef]

32. Bercu, G. The natural approach of trigonometric inequalities-Padé approximant. J. Math. Inequal. 2017, 11, 181-191. [CrossRef]

33. Bercu, G. Sharp bounds on the sinc function via the Fourier series method. J. Math. Inequal. 2019, 13, 495-504. [CrossRef]

34. Wu, S.-H.; Li, S.-G. Sharpened versions of Mitrinović-Adamović, Lazarević and Wilker's inequalities for trigonometric and hyperbolic functions. J. Nonlinear Sci. Appl. 2016, 9, 1-9. [CrossRef]

35. Wu, S.-H.; Srivastava, H.M. A weighted and exponential generalization of Wilker's inequality and its applications. Int. Trans. Spec. Funct. 2008, 18, 529-535. [CrossRef]

36. Chen, C.-P. Sharp Wilker and Huygens type inequalities for inverse trigonometric and inverse hyperbolic functions. Int. Trans. Spec. Funct. 2012, 23, 865-873. [CrossRef]

37. Chen, C.-P.; Cheung, W.-S. Wilker- and Huygens-type inequalities and solution to Oppenheim's problem. Int. Trans. Spec. Funct. 2012, 23, 325-336. [CrossRef]

38. Huang, W.-K.; Chen, X.-D.; Mao, X.-Y.; Chen, L.-Q. New inequalities for hyperbolic functions based on reparameterization. RACSAM 2021, 115, 3. [CrossRef]

39. Mortici, C. A Subtly Analysis of Wilker Inequality. Appl. Math. Comput. 2014, 231, 516-520. [CrossRef]

40. Nenezić, M.; Malešević, B.; Mortici, C. New approximations of some expressions involving trigonometric functions. Appl. Math. Comput. 2016, 283, 299-315. [CrossRef]

41. Zhu, L. Some new Wilker-type inequalities for circular and hyperbolic functions. Abstr. Appl. Anal. 2009, 2009, 485842. [CrossRef]

42. Wu, S.-H.; Debnath, L. A generalization of L'Hôspital-type rules for monotonicity and its application. Appl. Math. Lett. 2009, 22, 284-290. [CrossRef]

43. Malešević, B.; Lutovac, T.; Rašajski, M.; Mortici, C. Extensions of the natural approach to refinements and generalizations of some trigonometric inequalities. Adv. Differ. Equ. 2018, 2018, 90. [CrossRef]

44. Lutovac, T.; Malešević, B.; Mortici, C. The natural algorithmic approach of mixed trigonometric-polynomial problems. J. Inequal. Appl. 2017, 2017, 116. [CrossRef] [PubMed]

45. Lutovac, T.; Malešević, B.; Rašajski, M. A new method for proving some inequalities related to several special functions. Results Math. 2018, 73, 100. [CrossRef]

46. Malešević, B.; Rašajski, M.; Lutovac, T. Refinements and generalizations of some inequalities of Shafer-Fink's type for the inverse sine function. J. Inequal. Appl. 2017, 2017, 275. [CrossRef]

47. Rašajski, M.; Lutovac, T.; Malešević, B. About some exponential inequalities related to the sinc function. J. Inequal. Appl. 2018, 2018, 150. [CrossRef] [PubMed]

48. Banjac, B.; Makragić, M.; Malešević, B. Some notes on a method for proving inequalities by computer. Results Math. 2016, 69, 161-176. [CrossRef]

49. Zhu, L. New inequalities of Wilker's type for circular functions. AIMS Math. 2020, 5, 4874-4888. [CrossRef]

50. Wu, S.; Srivastava, H.M. A further refinement of a Jordan type inequality and its application. Appl. Math. Comput. 2008, 197, 914-923. [CrossRef]

51. Wu, S.; Srivastava, H.M.; Debnath, L. Some refined families of Jordan type inequalities and their applications. Integral Transform. Spec. Funct. 2008, 19, 183-193. [CrossRef]

52. Wu, S.; Debnath, L. Wilker-type inequalities for hyperbolic functions. Appl. Math. Lett. 2012, 25, 837-842. [CrossRef]

53. Wu, S.; Baricz, Á. Generalizations of Mitrinović, Adamović and Lazarević's inequalities and their applications. Publ. Math. Debr. 2009, 75, 447-458.

54. Bagul, Y.J.; Chesneau, C. Marko Kostić, On the Cusa-Huygens inequality. RACSAM 2021, 115, 29. [CrossRef]

55. Pinelis, I. L'Hospital rules for monotonicity and the Wilker-Anglesio inequality. Am. Math. Mon. 2004, 111, 905-909. [CrossRef] 
56. Chouikha, A.R. Sharp inequalities on circular and hyperbolic functions using Bernoulli inequality types. Rev. Real Acad. Cienc. Exactas FíSicas Nat. Ser. Matemáticas 2021, 115, 143.

57. Bagul, Y.J.; Chesneau, C. Some New Simple Inequalities Involving Exponential, Trigonometric and Hyperbolic Functions. CUBO Math. J. 2019, 21, 21-35. [CrossRef]

58. Becker, M.; Stark, E.L. On a hierarchy of quolynomial inequalities for tan x. Univ. Beograd. Publ. Elektrotehn. Fak. Ser. Mat. Fiz. 1978, 602-633, 133-138.

59. Zhu, L. A refinement of the Becker-Stark inequalities. Math. Notes 2013, 93, 421-425. [CrossRef]

60. Zhu, L. Sharp Becker-Stark-Type inequalities for Bessel functions. J. Inequal. Appl. 2010, 2010, 838740. [CrossRef]

61. Nenezić, M.; Zhu, L. Some improvements of Jordan-Stečkin and Becker-Stark Inequalities. Appl. Anal. Discret. Math. 2018, 12, 244-256. [CrossRef]

62. Rassias, T.M.; Andrica, D. (Eds.) Differential and Integral Inequalities. In Springer Optimization and Its Applications; Springer: Berlin/Heidelberg, Germany, 2019; Volume 151.

63. $\mathrm{Wu}, \mathrm{Y} . \mathrm{T}$; Bercu, G. New refinements of Becker-Stark and Cusa-Huygens inequalities via trigonometric polynomials method. RACSAM 2021, 115, 87. [CrossRef] 